Les femelles de races à viande accouplées avec des taureaux des mêmes races (race pure

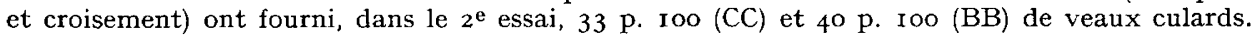

Sans tenir compte des variations d'expressivité du caractère qu'une répartition en 2 catégories ne permet pas d'apprécier, nous pouvons schématiser globalement les variations de fréquence du caractère culard dans la descendance des taureaux de ce type en :

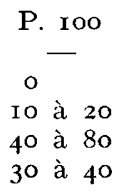

Ces résultats traduisent les variations d'expression du caractère suivant la présence du gène responsable ou de gènes modificateurs favorables. Ils soulignent, par ailleurs, l'intérêt d'une utilisation des femelles croisées pour la reproduction, du strict point de vue de la conformation des veaux tout au moins.

\title{
POSSIBILITÉS D'UTILISATION ET DE SÉLECTION DE TAUREAUX CULARDS EN VUE DU CROISEMENT DE PREMIÈRE GÉNÉRATION POUR LA PRODUCTION DE VEAUX DE BOUCHERIE
}

\section{Valls Ortiz, F. Menissier et B. Vissac. - Station de Génétique quantitative et appliquée C. N. R. Z., I. N.R.A., 78-Jouy-en-Josas (France).}

L'extension du croisement industriel et, plus généralement, des schémas de croisement utilisant des mâles de lignées spécialisées met en évidence l'intérêt des taureaux présentant le caractère culard. Depuis 1964 , dans 3 centres d'insémination artificielle nous avons étudié la descendance de 83 taureaux charolais normaux (4656 veaux) et 37 culards de la même race (1 862 veaux) comparés simultanément en croisement de première génération pour la production de veaux de boucherie élevés au pis et abattus entre 2 et 5 mois.

Les taureaux culards d'un échantillon de 6 reproducteurs de chaque type utilisés simultanément dans deux centres, fournissaient de I,2 à 7,2 p. Ioo de veaux culards selon le centre contre o p. Ioo pour les taureaux normaux. Le pourcentage de veaux ayant un phénotype intermédiaire était, pour ces mêmes centres, respectivement de Io,o et I7,3 p. Ioo pour les taureaux culards et 5,2 et 6,0 p. Ioo pour les normaux. Ces pourcentages de veaux culards issus des taureaux de même type variaient avec le développement musculaire des races maternelles soumises au croisement : I 4,0 p. Ioo dans le cas des races à viande contre 2,2 p. I0O pour les races rustiques et laitières.

La supériorité relative des veaux issus de taureaux culards par rapport aux produits de taureaux normaux était pour l'ensemble de l'échantillon de $+\mathrm{I}, 8$ p. roo sur le poids à la naissance, de $+\mathrm{I}, 6 \mathrm{p}$. Ioo sur le poids à 2 mois, de $4,5 \mathrm{p}$. Ioo sur le pointage de la valeur bouchère, et de $+6,2$ p. Ioo enfin sur la recette globale (produit des deux derniers critères).

La variabilité de la croissance et de la valeur bouchère est inférieure pour les reproducteurs culards par rapport aux normaux tant intra-taureaux $(\mathrm{P}<0,05)$ qu'entre taureaux $(\mathrm{P} \ll 0,05)$. Les coefficients d'héritabilité comparables sur le poids à la naissance $(0,18$ et 0,23$)$ pour les 2 types respectifs de taureaux sont significativement $(\mathrm{P}<0,05)$ plus faibles avec les taureaux culards sur le poids à 2 mois $(0,06$ contre 0,24$)$ et sur le pointage de valeur bouchère $(0,05$ contre o,39).

Ces résultats peuvent être interprétés soit par l'existence d'une structure génétique plus homogène chez les culards, soit par une moindre faculté d'adaptation aux conditions de milieu qui leur sont offertes dans les élevages notamment, en raison d'un poids plus élevé.

Si l'emploi des taureaux culards en croisement procure un avantage économique immédiat notable, la sélection à l'intérieur des taureaux de ce type risque de s'avérer moins efficace à plus long terme. Ces derniers résultats nécessitent une conformation sur un effectif plus important. 